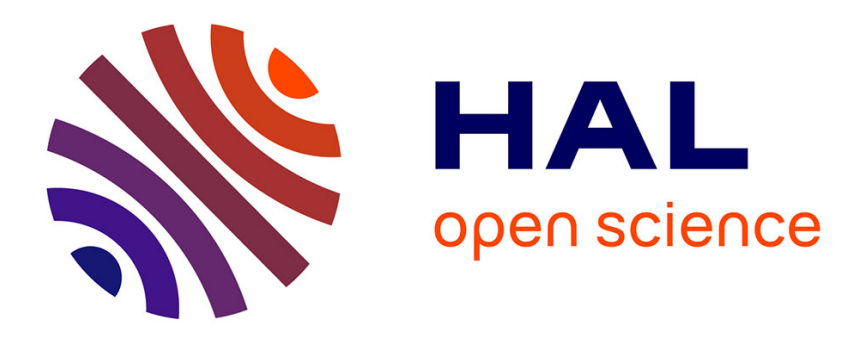

\title{
Sur la condition d'achromatisme dans les phénomènes d'interférence
}

\author{
A. Cornu
}

\section{To cite this version:}

A. Cornu. Sur la condition d'achromatisme dans les phénomènes d'interférence. J. Phys. Theor. Appl., 1882, 1 (1), pp.293-303. 10.1051/jphystap:018820010029300 . jpa-00237949

\section{HAL Id: jpa-00237949 https://hal.science/jpa-00237949}

Submitted on 1 Jan 1882

HAL is a multi-disciplinary open access archive for the deposit and dissemination of scientific research documents, whether they are published or not. The documents may come from teaching and research institutions in France or abroad, or from public or private research centers.
L'archive ouverte pluridisciplinaire HAL, est destinée au dépôt et à la diffusion de documents scientifiques de niveau recherche, publiés ou non, émanant des établissements d'enseignement et de recherche français ou étrangers, des laboratoires publics ou privés. 


\title{
SUR LA CONDITION D'AGHROMATISME DANS LES PHÉNOMÈNES D'INTERFÉRENCE ;
}

\author{
Par M. A. CORNU.
}

\section{I. - ThÉorie.}

Les expériences que j'ai entreprises pour l'étude des lois de la double réfraction circulaire, naturelle et magnétique, et que j'ai exposées précédemment ( $\left.{ }^{1}\right)$, m'ont amené à examiner et à résoudre une difficulté signalée par divers auteurs, et considérée même par M. Gouy $\left({ }^{2}\right)$ comme une objection à l'admirable explication du pouvoir rotatoire donnée par Fresnel.

En effet, j'ai utilisé le phénomène des franges latérales pour mesurer la différence entre les vitesses des ondes circulaires et la vitesse de l'onde ordinaire dans le quartz : or ce phénomène offre l'anomalie qui se rencontre dans tous les cas analogues, où l'on fait interférer des ondes ayant subi la double réfraction circulaire : les systèmes latéraux de franges sont écartés d'un peu plus du double de ce qu'une théorie approximative semble indiquer.

M. Billet, dans son excellent Traité d'Optique physique, signale ce désaccord et cite les chiffres qu'il a obtenus en répétant les expériences d'Arago, Fresnel et Babinet avec un quartz de $42^{\mathrm{mm}}$ (t. II, p. 242); ce passage résume parfaitement l'état de la question.

"... Ce quartz fait tourner le rayon moyen du spectre d'environ $42 \times 23^{\circ}=966^{\circ}$. Cela donne, à raison de $\mathrm{I} 80^{\circ}$ pour une onde de retard, un retard total d'environ 5,5 ondes. Ce devrait donc être sur la cinquième frange noire, là où le retard géométrique vaut 5,5 ondes, que devrait s'installer la frange centrale d'un système latéral, et la distance des centres des deux systèmes latéraux devrait être, par conséquent, de 22 franges simples ou de I I fois l'intervalle qui sépare deux franges semblables, deux

(') Journal de Physique, $2^{\mathrm{e}}$ séric, t. I, p. 1577.

(') Séances de la Société de Physique, année 1880 , p. 1?3.

J. de Phys., $2^{\circ}$ série, t. I. (Juillet 188..) 
noires par exemple. Il n'en est rien : celte distance dépasse le double de cette valeur théorique et atteint le chiffre de 24. "

L'expérience, faite avec un bloc unique de quartz, suivant le dispositif Arago-Fresnel, exige l'cmploi d'un polariseur et d'un analyseur; mais clle peut se répéter dans des conditions plus simples, arec la lumière naturelle, lorsque l'on emploie un biquart: à deux rotalions, comme dans l'expérience rappelée dans ma précédente Communication : l'anomalie est exactement la même. Avec les biquariz à axes croisés, que j’ai employés dans mes observations, les conditions théoriques sont également réduites au maximum de simplicité (1); la mème anomalie subsiste encore.

L'une de mes premières préoccupations a done été d'examiner ce désaccord apparent, non pas que la démonstration expérimentale de la loi à laquelle je suis parvenu pût en être infirmée (fondée sur la symétrie des deux systèmes latéraux, elle est indépendante de cette anomalie), mais pour ne pas laisser passer une difficulté touchant de si près le mode expérimental adopté.

Le résultat de ces recherches préliminaires, qui ont embrassé des cas divers et nombreux, se rísume en un théorème général dont roici l'énoncé :

Dans un système de franges d'interférences produites ̀̀ l'aille d'une lumière hétérogène ayant un spectre continu, il existe toujours une frange achromatique qui joue le rôle de frange centrale et qui se trouve au point du champ oì les radiations les plus intenses présentent une difjérence ale phase maximum ou minimum.

Ce thóorème, díduit d'abord de l'examen de cas très particuliers, est une simple conséquence de la constitution d'un système de franges d'interférences, de sorte qu'on peut en donner la démonstration indépendamment des phénomènes qu'il est destinś à expliquer.

Soit $b$ la distance des deux ouvertures (réelles ou fictives) qui livrent passage aux deux ondes réagissantes dont on observe

( ${ }^{1}$ L'emploi d’un polariscur ne sert qu'à éliminer le rayoa extraordinaire : le phénomène est risible sans analyseur; il le serait mème sans polariseur, mais le champ serait lavé de blanc et l'observation moins facile. 
l'interférence sur un tableau, à la distance $\mathrm{D}$, en un point situé à la distance $u$ du milieu géométrique du champ des franges rec¿ilignes.

Ces deux ondes, issues de la même source et d'égale intensité $\mathbf{A}_{\hat{\lambda}}^{2}$, ont une différence de phase $\stackrel{\varphi}{ }$, provenant de deus causes :

I $^{\circ}$ De la difference optique des chemins parcourus dans les divers milieux placés en avant des ouvertures : la différence de phase provenant de ce chef dépend de la couleur, c'est-à-dire de la longueur d'onde de la lumière emplosée; on la désignera, en géníral, par $\mathrm{F}(\lambda)$;

$2^{\circ}$ De la différence de distance des ouvertures au point $u \mathrm{du}$ tableau; la différence de phase correspondante est ćvidemment égale à $\frac{b u}{\lambda \mathrm{D}}$, d'où l'on conclut

$$
\varphi=F(\lambda)+\frac{b u}{\lambda \mathrm{D}}
$$

D'après la règle de Fresnel, l'intensité au point u sera

$$
I=A_{n}^{2}+A_{i}^{2}+2 A_{i} \cdot A_{2} \cos 2 \pi 0=4 A^{2} \cos ^{2} \pi \varphi \text {. }
$$

Avec la lumière blanche, on aura la superposition d'une infinité de scmblables systèmes, de sorte que l'intensitś en un point du champ sera représentée par la somme

$$
I=4 \pi A_{\%}^{2} \cos ^{2} \pi \varphi
$$

le signe $\Sigma$ renversé rappelani qu'on a à opérer tine sommation d'effets physiologiques et non pas une sommation de quantitís algébriques:

S'il existe une frange sensiblement achromatique, c'est-à-dire telle que toutes les couleurs (au moins les plus intenses pour l'œil) soient altérées dans la même proportion, elle doit se trouver en un point $u$, tel que la valeur de $\varphi$ soit indépendante de $\lambda$ : ce qui impose la condition analytique

ou bien

$$
\frac{c^{\prime r}}{d i}=0
$$

$$
F^{\prime}(\lambda)-\frac{b u}{\lambda_{1}^{2} D}=0
$$

Cette condition (qui suppose implicitcment que la variable $\lambda$ ne 
présente aucune discontinuité) signifie que la phase au point cherché passe par un maximum ou un minimum.

L'équation de condition ne contenant u qu'au premier degré, il existera toujours un semblable point définissant ainsi une ligne neutre ou achromatique, et il n'en existera qu'un seul : le théorème est donc démontré.

Propriétés de la ligne achromatique. - $1^{\circ} \mathrm{La}$ fonction $\mathrm{F}(\lambda)$ étant quelconque, la condition $\frac{d \mathfrak{o}}{d \lambda}=$ o est remplie d'une manière rigoureuse pour une certaine radiation $\lambda$, et d'une manière approximative seulement pour les autres; mais, avec les formes particulières de $F(\lambda)$ qu'on rencontre dans les expériences et dans les circonstances ordinaires d'observation, il arrive que, si celte condition est réalisée pour le rayon moyen du spectre (ou mieux pour le plus intense), elle est réalisée suffisamment pour toute l'étendue du spectre visible, les divergences sur les rayons extrêmes, c'est-à-dire les moins intenses, ne produisant que des colorations peu sensibles.

$2^{\circ}$ Ces divergences sont sensiblement annulées lorsque la valeur de $\varphi_{\text {p }}$ est voisine d'un nombre pair ou impair de fois la fraction $\frac{1}{2}$, parce que $\cos ^{2} \pi$ p passe alors par un maximum ou un minimum.

$3^{\circ}$ L'intensité de la lumière sur la ligne neutre est très approximativement égale à $4 \cos ^{2} \pi \varphi ̧ \zeta A_{\lambda}^{2}$, puisque la valeur de $\varphi$ est commune à toutes les radiations : elle peut prendre toutes les nuances de gris incolore comprises depuis le blanc parfait, valeur maximum égale à $4 \mathbf{K} \mathrm{A}_{\lambda}^{2}$ jusqu'au noir complet, valeur minimum égale à zéro.

$4^{\circ}$ La frange qui englobe cette ligne neutre présente le minimum de colorations; on peut l'appeler la frange achromatique; dans les cas extrêmes, cette frange achromatique est blanche ou noire (à centre blanc ou noir).

$5^{\circ}$ Lorsque la frange achromatique est noire ou blanche, elle est sensiblement frange centrale du système, car la ligne neutre qu'elle contient est une ligne de symétrie des colorations de cette frange; en effet, toutes les couleurs ont leur maximum ou minimum d'intensité sur la ligne neutre, car on a

$$
\frac{d \mathrm{I}}{d u}=4 \pi \frac{b}{\lambda \mathrm{D}} 3 \mathrm{~A}_{\mathrm{\lambda}}^{2} 2 \sin \pi \varphi \cos \pi \varphi=0 .
$$


Dans le cas général, au contraire, la frange achromalique est dissymétrique comme irisation.

$6^{\circ}$ Si l'expression de la phase contient un paramètre arbitraire, indépendant de $\lambda$ et de $u$, la condition $\frac{d \varphi}{d \lambda}=o$, et par suite la position de la ligne achromatique, en est indépendante. La variation continue de ce paramètre entraîne le déplacement continu des franges, mais n'altère pas la fixité de cette ligne achromatique qui reste toujours au milieu du système; la frange qui la traverse devient la frange achromatique et passe successivement par toutes les variétés indiquées ci-dessus.

Remarque. - L'analyse précédente montre l'inexactitude de la théorie adoptée jusqu'ici pour la détermination de la frange centrale; on cherchait en effet, comme l'indique la citation du début, le point du champ où la différence de phase d'une certaine couleur (rayon moyen) est nulle, $\varphi=0$, condition toute différente de celle qui se déduit de l'analyse du phénomène; aussi ce point ne correspond-il pas à la frange centrale apparente, puisqu'il varie lorsqu'on choisit une autre couleur.

L'erreur que l'on commettait provient d'une généralisation défectueuse de ce qu'on doit appeler une frange centrale. Dans le cas d'un système normal de franges (miroirs de Fresnel, trous d'Young, etc.), où la différence de phase se réduit à $\downarrow=\frac{u b}{\lambda \mathrm{D}}$, le milieu du champ est occupé par une frange qu'on a prise comme type des franges centrales; en ce point, $u=0$, la différence de phase esı nulle quelle que soit la couleur; cette condition est double et conduit à deux généralisations différentes dans le cas où la différence de phase est une fonction plus complexe de la longueur d'onde.

La première consiste à définir la frange centrale par la condition que la différence de phase soit nulle pour une couleur donnée (rayon moyen du spectre); mais cette définition, on l'a vu, ne répond pas au phénomène qu'on veut observer et conduit à des résultats en désaccord complet avec l'expérience.

La seconde, que je propose, consiste à définir, non pas en réalité une frange centrale et symétrique, ce qui n'est pas possible en général, mais la frange achromatique, celle qui présente le minimum d'irisation : on a vu qu'elle existe toujours et que même, 
dans certains cas, elle est très sensiblement centrale et sımétrique.

Il est facile de voir que cette définition correspond réritablement au phénomène que les physiciens ont en vue et qu'elle conduit aux résultats mèmes de l'expérience.

Je choisirai précisément l'exemple cité par M. Billet. Il suffit, pour le traiter, de connaître la valeur particulière de la phase? dans l'expérience d'Arago-Fresnel. On trouve aisément (1) pour l'expression de lïntensité en un point quelconque $u$ du champ

$$
\mathbf{I}=\hat{i} \mathrm{~A}_{\Lambda}^{2} \cos ^{2}(\Omega \div \approx) \cos ^{2} \pi \dot{y},
$$

on l'équivalent

$$
I=A_{\lambda}^{2}\left[\cos (\Omega+\alpha+\pi)+\cos (\Omega-\alpha-\pi+)_{1}^{2},\right.
$$

$\boldsymbol{\Omega}$ étant l'angle des sections principales du polariseur et de l'analyseur et o l'angle dont le bloc de quartz fait tourner le plan de polarisation de la radiation $\lambda$. On sait, d'après la loi de Biot, que cet anğle est proportionnel à la longueur $e$ du quartz et à peu près en raison inverse du carré de $\lambda$

$$
\alpha=\frac{\mathbf{H} e}{i ! 2} .
$$

Je ne m'arrèterai pas à dímontrer que ces formules rendent compte des trois systèmes de franges qu'on obscrve arec la lumière blanche : il suffit de remarquer que les deux systèmes latéraux ont respectivement pour équations

$$
I=\Lambda{ }^{2} \cos ^{2}(\Omega-\xi-\pi), \quad I=\Lambda_{\alpha}^{2} \cos ^{2}(\Omega+\pi-\pi),
$$

qu'on obtiendrait directement par la considératicn des ondes à vibration circulaire. La forme de ces équations est naturellement celle qui a été discutée plus haut, et la valeur de tọ est précisément l'argument du cosinus: on remarquera, en passant, le para-

(') Les deux faisceaux incidents polariśs ont pour ampliiude $I_{\lambda}$; leur vibration, faisant l'angle $\Omega$ ares un ax $\_$arbitraire, fait l'angle $\Omega-i \sim$ après la sortie du quartz : la composante conserrée par l'analyseur dont la section est dirigéc suivant l'axe arbitraire sera $\Lambda_{\lambda} \cos (\Omega+x)$. La difŕrence de phase due à l'obliquité sur le; ouvertures sera, comme plus haut, $4=\frac{b u}{\lambda \mathrm{D}}$. La règle de Fresnel donne l'cxpression ci-dessus. 
mètre arbitraire $\Omega$ indépendant de $u$ et de $\lambda$ dont il a été qucstion et qui explique le déplacement des franges par la rotation de l'analyseur, leụr variation continue de colorations, et malgré cela la fixité de la frange centrale apparente périodiquement blanche ou noire.

La ligne achromalique est définie par la condition

$$
\frac{d x}{d \lambda}=\pi \frac{b \prime}{\lambda^{2} \nu}=0
$$

qu'on peut ćcrire

$$
\frac{\mathrm{I}}{\pi} \lambda \frac{d x}{d \lambda}=\frac{u}{\frac{\lambda \mathrm{D}}{b}}=0 .
$$

Le dénominateur de $u$ représente la largeur X d'une frange (définie par la condition $\psi=\mathrm{I}$ ). Si maintenant on substitue la dérivée de a, tirée de la loi de Biot,

$$
\frac{d x}{d \lambda}=-\frac{2 \mathrm{H} e}{\lambda^{3}}, \quad \text { d'où } \quad \lambda \frac{d x}{d \lambda}=-\frac{2 \mathrm{H} e}{\lambda^{4}}=-2 x
$$

il vient, en définitive,

$$
u=\frac{2 x}{\pi}=0
$$

La ligne achromatique est donc distante du milieu du champ d'un nombre de franges égal au double du nombre de fois suivant lequel $180^{\circ}$ est compris dans l'angle de rotation du quartz.

C'est le double de ce que prévoyait la théorie défectueuse, et c'est précisément ce que donne l'observation.

L'anomalie prétendue n'existe donc nullement, et le phénomène cst une conséquence de la loi de Biot (').

( ') L'emploi d'une formule empirique plus exacte permet de serrer encore de plus près l'expérience : ainsi, en déterminant l'exposant $\varepsilon$ de $\lambda(\varepsilon=-2$ dans la loi de Biot) par la condition de représenter le mieux possible les résultats de MM. Soret et Sarasin (Comptes rendus des séances de l'Académie des Sciences, t. LXXXIII, p. $8 \mathrm{r} 8$ ) dans l'étendue du spectre visible, on trouve $\varepsilon=-2, \mathrm{r} 3:$ la simplicité de la démonstration n'en est pas altérśe, mais le facteur 2 est remplacé par 2,r.3. Ce chiffre, suĐstitué dans des données numériques de M. Billet, donne 


\section{II. - Expériences de vérification.}

On réalise aisément par l'expérience les conditions développées ci-dessus : voici les principaux phénomènes que j’ai pu montrer par projection ( 1 ).

\section{Déplacement de la frange achromatique à l'aide d'un prisme d'angle variable.}

Si l'on projette un système de franges sur un écran blanc, et qu'on interpose un diasporamètre de Boscowitch ou de Brewster, chaque lumière simple, par conséquent chaque système monochromatique de franges, est déplacé d'une quantité dépendant de sa longueur d'onde, à cause de la dispersion du prisme; si l'on fait croître progressivement l'angle du prisme, on voit les franges se déplacer, et la coloration se modifier; bientôt la symétrie initiale de la frange centrale blanche se présente autour de la frange sombre contiguë, qui devient alors complètement noire : l'augmentation de l'angle du prisme continuant, la frange centrale noire s'irise dissymétriquement, et la frange claire contiguë devient bientôt frange centrale blanche, et ainsi de suite : si bien que la frange achromatique paraît suivre le mouvement de translation des franges, mais en allant, en apparence, un peu moins vite que le déplacement général.

Le calcul permet de prévoir ce résultat : en effet, si l'on désigne, comme plus haut, par $u$ la distance au milieu du champ, où la phase est $\varphi$, on a pour un système de franges normal

$$
\varphi=\frac{b u}{\lambda \mathrm{D}}
$$

pour la distance des franges achromatiques des deux systèmes le nombre

$$
\text { I } \times 2, \text { r } 3=23,4,
$$

résultat aussi voisin que possible du chiffre observé, 24 , eu égard à l'approximation qu'on est en droit d'attendre du phénomène, qui n'est appréciable qualitativement qu'à une frange près.

( ${ }^{1}$ ) Avec le concours empressé de M. J. Duboscq, qui a bien voulu faire tailler diverses pièces de quartz, spécialement en vue d'exécuter plus facilement ces expériences. 
Si ce point est déplacé par la réfraction du prisme, il se trouvera en un point $x$ tel que

$$
x=u+c_{\gamma},
$$

$c_{\lambda}$ étant le déplacement que le prisme produit sur la radiation $\lambda$.

On peut considérer, dans une première approximation, que $c_{\lambda}$ est proportionnel à l'angle variable du prisme A et de la forme

$$
c_{\lambda}=\mathbf{A}(p-q \lambda),
$$

$p$ et $q$ étant deux constantes positives, parce que la déviation des radiations à courte longueur d'onde est plus grande que celle des radiations à grande longueur d'onde; d'où l'on conclut, pour la détermination du point $x$ où la phase est p̣, l'expression

$$
\varphi^{\prime}=b \frac{x-c_{\lambda}}{\lambda \mathrm{D}}=b \frac{x-\mathrm{A} p-\mathrm{A} q \lambda}{\lambda \mathrm{D}}=b \frac{x-\mathrm{A} p}{\lambda \mathrm{D}}+b \frac{\mathrm{A} q}{\mathrm{D}} .
$$

La position $x$ de la frange achromatique est donnée par la dérivée de $\varphi$ par rapport à $\lambda$, égalée à zéro,

$$
x-\mathbf{A} p=\text { o. }
$$

On voit que le déplacement de la frange achromatique est proportionnel à l'angle du prisme et que la phase correspondante est une constante $\frac{b \mathrm{~A} q}{\mathrm{D}}$ indépendante de $\lambda$, ce qui donnerait un achromatisme parfait, si l'expression adoptée pour $c_{\lambda}$ était rigoureuse.

Les franges centrales blanches successives correspondent aux valeurs de $\frac{b \mathrm{~A} q}{\mathrm{D}}$ égales à $\mathrm{o}, 1,2$, etc.; les franges noires, aux valeurs intermédiaires $\frac{1}{2}, \frac{3}{2}, \frac{5}{2}$, etc.

L'analyse de cette expérience si simple est très instructive et mérite d'être détaillée par le lecteur désireux de se rendre un compte exact des conditions d'achromatisme dans les phénomènes d'interférence.

La projection est très brillante, même avec la lumière Drummond, si l'on produit les franges avec le compensateur de Babinet.

$2^{\circ}$ Réalisation d'une variation de la phase commune à toutes les radiations.

C'est le cas indiqué sous le numéro $6^{\circ}$ dansl'Étude des propriétés 
de la ligne achromalique (p. 297) et rappelé plus loin (p. 298) à propos des trois systèmes de franges.

On forme un parallélepipède arec deux prismes de quartz de rotation contraire, taillés à $30^{\circ}$, l'axe étant perpendiculaire à l'une des faces, et on les colle ensemble, par la face oblique, de manière que les deux faces parallèles soient perpendiculaires à l'axe. Ce parallélépipède donne dans la lumière polariséc un beau système de franges dont le lieu apparent est au milieu du bloc.

La frange centrale correspond au point où les épaisseurs de quartz sont égales et se compcnsent comme pouvoir rotatoire. Il est facile de voir, par une formule analogue à cclle qui est donnée plus haut, que la différence de phase contient, comme paramètre variable, l'angle que fait le polariseur avec l'analyseur.

Il en résulte que la condition $\frac{d e}{d \lambda}=0$ est indépendante de cet angle, c'est-à-dire que la ligne achromatique est fixe.

L'expérience montre en effel que la rolation continue de l'analyseur altère profondément les teintes des franges; que ces franges paraissent se déplacer d'un mouvement continu; mais la frange achromatique reste toujours fixe au centre du systìme, passant périodiquement à l'état de frange centrale blanche et noire alternativement.

\section{$3^{\circ}$ Projection des trois systèmes de franges d'Arago et Fresnel.}

Les systèmes latéraux, dans l'expérience des trois systèmes de franges, présentent cette propriété curieuse, isolée dans l'expérience précédente, de paraître, par rolation de l'analyseur, mobiles comme les filets d'une vis sans fin, et, en réalité, de rester en place. On réussit à projeter ce phénomène avec la lumière Drummond, cn modifiant légèrement l'expérience primitive d'Arago avec le dispositif suivant. On prend un prismc biréfringent en quartz d'un angle extrêmement petit, intermédiaire entre l'angle des plus faibles et celui qu'on adopte pour le compensateur de Babinet. Cet appareil, placé entre un polarisatcur et un analyseur, donne un système de franges très serrées dont le lieu apparent est au milieu de l'épaisseur du prisme. Entre lc prisme et l'analyseur on interpose un bloc de

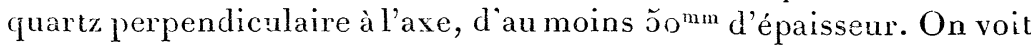


A. HURION. - ICHROMITISIE.

803

alors les trois systèmes dont la théorie a été donnée ci-dessus. On reconnaît dans les trois systèmes la fixité de la frange achromatique, malgré les mourements apparents centrifuges ou centripètes des systèmes latéraux. 